\section{Consumo televisivo y autopercepción en las personas mayores}

\section{Television consumption and elderly's self-perception}

\author{
Marga Vives Barcelón,** \\ Lydia Sánchez-Prieto ${ }^{2}$ \\ Marta Torres Olías ${ }^{3}$ \\ 1. Profesora contratada doctora interina. Universitat de les Illes Balears. \\ Palma de Mallorca. Illes Balears. España. \\ 2. Investigadora predoctoral FPI. Universitat de les Illes Balears. Palma de Mallorca. \\ Illes Balears. España. \\ 3. Educadora Social. Instituto Mallorquín de Asuntos Sociales (IMAS). \\ Palma de Mallorca, Illes Balears, España. \\ *Autor para correspondencia. \\ Correo electrónico: marga.vives@uib.es (Marga Vives Barceló).
}

\section{RESUMEN}

El presente trabajo analiza la posible contribución de la publicidad televisiva al fomento de los estereotipos en el colectivo de personas mayores.

Se recoge en este artículo, la revisión de las principales investigaciones realizadas en las que se plantea cómo la televisión articula a través de la publicidad la imagen de las personas mayores además de contar con la opinión de una muestra seleccionada de 40 personas mayores donde se ha tenido en cuenta el factor de la institucionalización. Los resultados confirman que no hay diferencias entre el consumo de horas de televisión y la autopercepción, pero sí en autovaloración positiva en personas no institucionalizadas $(p=0,46)$ y en deterioro de proceso cognitivo $(p=0,000)$.

Al mismo tiempo, las personas institucionalizadas afirman que la vejez impide realizar actividades que a uno le gustan $(p=0,027)$.

En conclusión, la institucionalización supone una variable clave en la percepción sobre las personas mayores, posiblemente influenciada por el contacto con sus familias y una mayor autonomía.

PALABRAS CLAVE: Personas mayores, publicidad, medios de comunicación, autoevaluación, institucionalización y calidad de vida.

\section{ABSTRACT}

This work pretends to study the possible contribution of the television advertising to increase the stereotypes about the elderly people collective.

This article makes a review about the main investigations done in which is considered how the television shows through the advertising the image of the elderly people as well as present the opinion of a sample selected composed by forty elderly persons keeping in mind the institutionalization as a factor.

Results shows no differences between hours of consumption of television and self-perception, but positive differences between positive auto-evalutation in non-institutionalization persons $(\mathrm{p}=0,46)$ and with cognitive process deterioration $(p=0,000)$. Institutionalization persons affirm that old age block to realize activities that one likes $(p=0,027)$. In conclusion, the institutionalization of elderly persons is a key factor in the perception of the old age, probably influence by greater autonomy and by contact with their families.

KEYWORDS: Older people, Advertising, Mass media, Self-evaluation Institutionalization \& Quality of life.

\section{- INTRODUCCIÓN}

\section{Institucionalización de personas mayores}

Uno de los cambios sociales que más describe nuestro contexto es el progresivo envejecimiento universal de la población ${ }^{1}$. Al descenso de la tasa de natalidad hay que añadir el hecho de que la esperanza de vida es cada vez mayor; vivimos más tiempo y en mejores condiciones físicas ${ }^{2}$. La incorporación de la mujer al mercado laboral reduce potencialmente el número de cuidadoras informales. Por tanto, resulta innegociable la necesidad de habilitar recursos sociosanitarios, como ocurre con las residencias, tanto públicas como privadas, convirtiéndose incluso en imprescindibles ${ }^{3}$.

La vida en la residencia proporciona a las personas mayores paz, sosiego y bienestar, aunque en un segundo análisis se puede apreciar ambigüedad en los sentimientos de bienestar, y ese vivir bien puede explicarse como que la resignación es lo que les ayuda a encontrarse bien ${ }^{4}$. Siguiendo las directrices de la Organización Mundial de la Salud, la calidad de vida ideal comprende un lugar o hábitat satisfactorio, amigos, familia acogedora y comprensiva, desahogo económico, sensación de felicidad, ausencia de enfermedad, buen estado intelectual y ausencia de enfermedades 5 . Sin embargo, con frecuencia, los centros residenciales en los que ingresan las personas mayores suelen estar alejados de su residencia habitual, el cambio forzoso de entorno suele provocar un sentimiento de desarraigo, que a menudo merma la salud y la sensación de satisfacción con la vida de la persona mayor ${ }^{6}$. Resulta imprescindible, por parte de las residencias, cumplir con objetivos y funciones que enfaticen el carácter fundamentalmente "social" de los mismos, lo que garantizará su calidad de vida y bienestar personal y social en el centro ${ }^{6}$.

\section{Las personas mayores y la publicidad televisiva}

La televisión funciona como un entretenimiento común en el colectivo de personas mayores ${ }^{1,7,8}$; así se recoge en el informe del IMSERSO 
$(2011)^{9,10}$, donde se expresa que, de los siete millones y medio de personas mayores registradas actualmente en España, casi un 17\% de la población puede verse influenciada por los medios de comunicación. Sin duda, las personas mayores son un importante segmento de mercado, puesto que son grandes consumidores de los medios ${ }^{2}$, especialmente la televisión. Los mensajes que emiten los medios de comunicación influyen en los esquemas culturales de los diferentes grupos sociales, lo que causa confusión en el público que los recibe? . Por lo tanto, la publicidad o los mensajes acaban imponiendo criterios morales, éticos, culturales y estéticos $^{11}$. El impacto actitudinal se ve favorecido si además aparecen personajes similares a los propios espectadores ${ }^{12}$.

Se debe remarcar que hace relativamente poco tiempo que los medios de comunicación han empezado a valorar y a introducir la imagen de las personas mayores en la publicidad; de hecho, se trataba de un colectivo que tradicionalmente estaba excluido del foco de la atención mediática ${ }^{13,14}$. Además, los medios de comunicación, aferrados al pasado, siguen haciendo uso de una fotografía antigua para mostrar a las personas mayores ${ }^{15}$; se refieren con frecuencia a las personas mayores como si se tratara de un grupo social homogéneo, como si todos sus miembros fueran iguales ${ }^{16}$. La invisibilidad es seguramente el ejercicio más común de violencia simbólica en los medios de comunicación cuando se habla de grupos desfavorecidos, lo que coincide con la situación del sector de mayores ${ }^{1}$. $\mathrm{O}$ bien, las personas mayores reciben referentes o modelos culturales aplicables a ellos mismos, fundamentalmente de los productos comerciales difundidos por los medios de comunicación de masas; modelos, en muchos casos, no aceptados por este grupo de población ${ }^{17}$. En este sentido, los medios de comunicación tienen un papel esencial para este colectivo de edad, lo que debería reflejarse tanto en la presencia temática de esta franja de edad y sus intereses como en su representación en los medios, que, si bien en ocasiones es positiva, suele ser mayoritariamente negativa en los medios de comunicación ${ }^{13}$.

Concretamente, se identifica la ausencia de la figura del mayor en la publicidad $^{18}$; tan solo un $4,5 \%$ de los personajes que aparecen en los anuncios publicitarios tienen 60 años o más ${ }^{1}$, concluyendo en la idea de que las personas mayores están infrarrepresentadas en los anuncios televisivos ${ }^{2}$. Por otro lado, se manifiesta también un desequilibrio en los magazines radiofónicos; se registra una presencia menor de las personas mayores de 65 años o más $(18,2 \%)$ respecto al resto de población y una presencia de solo el $4,65 \%{ }^{13}$.

El desarrollo de discriminaciones y de actitudes perjudiciales podrá arraigar los estereotipos, lo que fomenta la aparición de edadismos ${ }^{19}$. El problema es que la descripción negativa de la vejez en los medios de comunicación no solo puede afectar a la manera en que las personas mayores se perciben, sino que, además, puede tener un efecto opresivo en su bienestar mental ${ }^{20}$; también una representación inadecuada o insuficiente en la publicidad de las personas mayores afecta a la imagen y actitudes sociales que los otros tienen de este grupo ${ }^{21}$. La infraestimación de las capacidades físicas y mentales de las personas mayores puede favorecer una prematura pérdida de independencia, una mayor discapacidad, mayores índices de depresión y una mortalidad anticipada ${ }^{19}$. Las imágenes estereotipadas de las personas mayores han servido para definir los comportamientos considerados apropiados para este colectivo $^{22}$, pero es cierto que las personas mayores quieren ser tratadas sin diferencias por cuestión de edad ${ }^{23}$.

Uno de los objetivos de la inclusión de las personas mayores en la sociedad de la información debería ser lograr una calidad de vida suficiente en su vejez, que pueda permitir a las personas mayores una vida más activa y participativa y puedan servir de instrumentos esenciales en el fomento de su participación cívica ${ }^{24}$.

\section{- OBJETIVOS}

El objetivo general es analizar la percepción que tienen las personas mayores de la imagen televisiva que se emite del colectivo de personas mayores. Como objetivos específicos, se pretende comprobar si esa percepción sobre la imagen del mayor varía entre personas institucionalizadas y no institucionalizadas. De igual modo, también valorar si existen diferencias entre personas institucionalizadas y no institucionalizadas en referencia al consumo televisivo (número de horas).

Por consiguiente, se plantean una serie de hipótesis de trabajo, divididas entre aquellas que hacen referencia a la imagen y la televisión ( $\mathrm{H} 1$ y H2), las relativas al consumo televisivo $(\mathrm{H} 3)$ y las que abordan las diferencias entre personas mayores institucionalizadas y no institucionalizadas referentes al consumo y percepción de la vejez (H4):

H1: un mayor consumo televisivo se relaciona de forma significativa con una autoimagen más negativa de la vejez.

$\mathrm{H} 2$ : un mayor consumo televisivo se relaciona de forma significativa con una imagen más estereotipada de la vejez.

H3: existe un mayor consumo televisivo por parte de personas mayores institucionalizadas en comparación con personas mayores no institucionalizadas.

H4: existen diferencias significativas entre el colectivo de personas institucionalizadas y no institucionalizadas en cuanto a una percepción negativa de la vejez.

Para valorar el consumo televisivo se contabilizaba 6 horas diarias.

\section{- METODOLOGÍA}

La realización del estudio se inició con un análisis documental en las principales bases de datos científicas en ciencias sociales (ISI Web of Knowledge, SJR, SCOPUS ESBCO y Science Direct). En el protocolo de búsqueda se utilizaron las palabras clave "Elder people", "Older people", "Institutionalization", "mass media” y "advertisement"; así como ecuaciones de búsqueda en inglés y en español.

Los criterios de inclusión de los documentos científicos respondieron a criterios temáticos (publicidad televisiva, autopercepción y personas mayores), criterios temporales (estudios comprendidos entre 2007 y 2017) y características de la muestra (personas mayores de 65 años priorizando población española).

La muestra seleccionada para tal estudio fueron personas mayores de 65 años residentes en Palma de Mallorca, con capacidades cognitivas suficientes para poder responder a los cuestionarios. De igual manera, se seleccionó solo a aquellas personas mayores que disponían de una capacidad visual que les permitiera ver y analizar los anuncios publicitarios. Todos los participantes firmaron un consentimiento informado sobre el cuestionario y el tratamiento y análisis de los datos obtenidos.

Dicha muestra estuvo formada por 40 personas, divididas en dos grupos (institucionalizados y no institucionalizados); la muestra de población institucionalizada fue seleccionada de la Llar d'Ancians, la residencia de referencia en Illes Balears; las personas mayores no institucionalizadas fueron seleccionadas en los centros de día de Casal del Jonquet y del Casal de la Soledat, dos centros próximos a la Llar d'Ancians y que permiten que no haya diferencias en cuanto a lugar de residencia entre las dos muestras.

Las personas han participado tras la firma de un consentimiento informado, que cumple con los criterios definidos en la LOPD 15/1999, y conocían la capacidad de ejercer los derechos ARCO (acceso, rectificación, cancelación u oposición). 
La evaluación de la percepción se estableció a partir de un cuestionario realizado con base en la bibliografía revisada, así como de instrumentos psicométricos de percepción de la imagen en los medios. El cuestionario se estableció a partir de los siguientes bloques de análisis, agrupados de la siguiente manera: a) datos sociodemográfi$\cos , b$ ) autopercepción, $c$ ) percepción sobre el colectivo de personas mayores, d) uso (y consumo) de la televisión, e) opinión sobre la aparición de personas mayores en televisión y f) influencia de la televisión en personas mayores.

\section{- RESULTADOS}

\section{Descripción de la muestra}

El conjunto de la muestra está formado por 20 personas institucionalizadas y 20 no institucionalizadas. La media de edad es de 78,35 años (desviación típica 9,04); un 57,5\% son mujeres y un 42,5\%, hombres. Un $77,5 \%$ son viudos $(60 \%)$ o solteros $(17,5 \%)$; con hijos $(67,5 \%)$ con los que tienen contacto $(92,6 \%)$ varios días a la semana $(48,1 \%)$; principalmente en persona $(51,9 \%)$ o a través de llamadas por teléfono $(25 \%)$. Se trata de una muestra con ingresos inferiores a $800 €(75 \%)$, casi la mitad sin estudios $(47,5 \%)$ o estudios de educación primaria $(32,5 \%)$; mayoritariamente dedicados al sector servicios en sus profesiones (50\%); sin grado de dependencia (95\%) ni problemas de movilidad $(72,5 \%)$, pero sí con problemas de salud desde hace años $(67,5 \%)$.

La tabla 1 aporta más información detallada sobre la muestra en variables de edad, estado civil y enfermedades relacionadas con su institucionalización o no. Las enfermedades se han agrupado a partir de las clasificaciones más comunes descritas en la bibliografía consultada.

Las hipótesis de trabajo se dividen en aquellas que hacen referencia a la imagen y la televisión ( $\mathrm{H} 1$ y $\mathrm{H} 2$ ), al consumo televisivo $(\mathrm{H} 3)$, y las diferencias entre personas mayores institucionalizadas y no institucionalizadas referentes al consumo y percepción de la vejez (H4).

\section{Autoimagen y estereotipos (H1 y H2)}

Para evaluar la autoimagen se realizó un bloque de preguntas relacionadas con el sentimiento de edad, el significado que tiene la edad, las aportaciones que pueden realizarse como persona mayor de 65 ańos, adjetivos definitorios (basados en la bibliografía) sobre la vejez y sentimiento de pérdida de habilidades.

La tabla 2 resume las relaciones significativas que hay entre consumo de televisión (6 horas o más)* y la autoimagen como persona de más de 65 ańos por muestra total y submuestras (institucionalizada y no institucionalizada). Los resultados muestran que no existen diferencias significativas entre consumo de horas de televisión y autopercepción. Solo se observan diferencias significativas en la muestra de no institucionalizados en cuanto a una autovaloración más positiva $(p=0,46)$; es decir, que las personas mayores no institucionalizadas puntúan de forma más positiva en ítems relacionados con su autoimagen.

Respecto a los estereotipos, se consultó sobre la percepción como colectivo homogéneo, pérdida de realización de actividades por el hecho de envejecer y si la sociedad tiene en cuenta a las personas mayores. Los resultados no muestran diferencias significativas entre el grupo institucionalizado y el no institucionalizado según el consumo de televisión; es de destacar que todos los institucionalizados consultados han confirmado que se trata de un colectivo heterogéneo (tabla 3).

\section{Consumo televisivo e institucionalización (H3)}

La muestra seleccionada presenta un perfil de consumo de televisión de manera diaria (90\%). En general, las mujeres de la muestra suelen

*Este target de edad dedica diariamente $4 \mathrm{~h} 40$ ' a ver la televisión (56 minutos más que el conjunto de la población). El $92 \%$ de la población mayor de 65 años ve la televisión todos los días, lo que les sirve para cubrir tres objetivos: información, ocio y evitar la soledad ${ }^{13}$.

Tabla 1. Descripción de la muestra

\begin{tabular}{|c|c|c|c|c|}
\hline & & No institucionalizado & Institucionalizado & Total \\
\hline & Mujer & $25,0 \%$ & $32,5 \%$ & $57,5 \%$ \\
\hline Género & Hombre & $25,0 \%$ & $17,5 \%$ & $42,5 \%$ \\
\hline & Total & $50,0 \%$ & $50,0 \%$ & $100,0 \%$ \\
\hline & Viudo/a & $27,5 \%$ & $32,5 \%$ & $60,0 \%$ \\
\hline & Soltero/a & $10,0 \%$ & $7,5 \%$ & $17,5 \%$ \\
\hline Estado civil & Casado/a & $2,5 \%$ & $5,0 \%$ & $7,5 \%$ \\
\hline & Separado/a & $5,0 \%$ & $2,5 \%$ & $7,5 \%$ \\
\hline & Total & $50,0 \%$ & $50,0 \%$ & $100,0 \%$ \\
\hline & Enfermedades óseas y musculares & $35,0 \%$ & $17,9 \%$ & $42,8 \%$ \\
\hline & Enfermedades del sistema nevioso & $3,6 \%$ & $10,7 \%$ & $14,3 \%$ \\
\hline Enfermedades & Enfermedades cardiovasculares y respiratorias & $7,1 \%$ & $10,7 \%$ & $17,8 \%$ \\
\hline & $\begin{array}{l}\text { Otras enfermedades (dermatológicas, urológicas, } \\
\text { oftalmológicas, demencias, cáncer)a }\end{array}$ & $10,8 \%$ & $10,8 \%$ & $21,6 \%$ \\
\hline & Total & 56,5 & $43,5 \%$ & $100 \%$ \\
\hline
\end{tabular}

a Se han agrupado al no superar ninguna de ellas el 3,6\% de la muestra. 
Tabla 2. Autoimagen y consumo (en horas) de televisión según institucionalización o no

\begin{tabular}{|c|c|c|c|c|c|}
\hline & Institucionalización & N & Valor & GI & Sig. asintótica (bilateral) \\
\hline \multirow{3}{*}{$\begin{array}{l}\text { Autopercepción } \\
\text { de la edad }\end{array}$} & Sí & 20 & 0,137 & 2 & 0,934 \\
\hline & No & 20 & 3,750 & 2 & 0,153 \\
\hline & Total & 40 & 1,708 & 2 & 0,426 \\
\hline \multirow{3}{*}{$\begin{array}{l}\text { Significado que } \\
\text { tiene la edad }\end{array}$} & Sí & 20 & 5,600 & 4 & 0,231 \\
\hline & No & 20 & 11,518 & 4 & 0,210 \\
\hline & Total & 40 & 1,495 & 4 & 0,828 \\
\hline \multirow{3}{*}{$\begin{array}{l}\text { Autopercepción } \\
\text { de ser útil }\end{array}$} & Sí & 20 & 0,606 & 2 & 0,739 \\
\hline & No & 20 & 1,250 & 2 & 0,535 \\
\hline & Total & 40 & 0,016 & 2 & 0,992 \\
\hline \multirow{3}{*}{ Autoimagen } & Sí & 20 & 7,556 & 5 & 0,182 \\
\hline & No & 20 & 8,021 & 3 & 0,046 \\
\hline & Total & 40 & 11,781 & 5 & 0,038 \\
\hline \multirow{3}{*}{$\begin{array}{l}\text { Autopercepción } \\
\text { de habilidades } \\
\text { funcionales }\end{array}$} & Sí & 20 & 0,000 & 1 & 1,000 \\
\hline & No & 20 & 0,580 & 2 & 0,748 \\
\hline & Total & 40 & 0,621 & 2 & 0,733 \\
\hline
\end{tabular}

Tabla 3. Percepción del colectivo de personas mayores de 65 años por horas de consumo de televisión

\begin{tabular}{|l|c|c|c|c|c|}
\hline & Institucionalización & N & Valor & GI & Sig. asintótica (bilateral) \\
\hline \multirow{2}{*}{$\begin{array}{l}\text { Colectivo } \\
\text { homogéneo }\end{array}$} & Sí & 20 & - & - & - \\
\hline & No & 20 & 2,143 & 1 & 0,143 \\
\hline $\begin{array}{l}\text { Pérdida de } \\
\text { actividades por } \\
\text { raźn de edad }\end{array}$ & Total & 40 & 2,049 & 1 & 0,152 \\
\hline \multirow{2}{*}{$\begin{array}{l}\text { Percepción de la } \\
\text { sociedad }\end{array}$} & No & 20 & 2,857 & 1 & 0,091 \\
\hline
\end{tabular}

Gl: grados de libertad.

consumir entre 5 horas $(21,7 \%)$ o 3 o 4 horas $(17,4 \%)$, respectivamente, mientras que los hombres consumen entre 3 horas $(29,4 \%)$ o 1 o 2 horas (17,6\%), respectivamente. Revisando el número de horas de consumo televisivo entre personas mayores institucionalizadas y las que no, tal como muestra la tabla 4, no existen diferencias significativas entre el hecho de estar institucionalizado o no y el consumo de horas de televisión. Finalmente, se incluyen en la descripción variables como el grado de dependencia (si se tiene), la movilidad (necesidad de apoyo con bastón, silla de ruedas o ningún apoyo para moverse), las dificultades para moverse (sin esfuerzo, con esfuerzo o con necesidad de otra persona) y habilidades funcionales (tales como reconocer si han recibido correctamente el cambio de una compra o utilizar adecuadamente el transporte). Solo en las habilidades funcionales aparecen diferencias significativas.
Diferencias entre personas mayores institucionalizadas y no institucionalizadas respecto al consumo y percepción de la vejez (H4)

Las personas participantes han afirmado, en un $97,5 \%$, que les gusta la televisión; no obstante, no se han apreciado diferencias significativas entre el hecho de estar institucionalizado o no con el número de horas de consumo televisivo $(p=0,196)$; sí aparece una relación significativa entre el hecho de estar institucionalizado y la percepción de que el envejecimiento impide realizar actividades que a uno le gusta hacer $(p=0,027)$. En este sentido, las personas institucionalizadas responden más afirmativamente a esta pregunta, mientras que las no institucionalizadas, en un $68,3 \%$ creen que el envejecimiento no impide realizar actividades que a uno le gustan. 
Tabla 4. Consumo televisivo según sexo y perfil de autonomía

\begin{tabular}{|c|c|c|c|c|c|}
\hline & & N & Valor & GI & Sig. asintótica (bilateral) \\
\hline & Sexo & 40 & 7,679 & 9 & 0,567 \\
\hline \multirow{3}{*}{$\begin{array}{l}\text { Colectivo } \\
\text { homogéneo }\end{array}$} & Institucionalización & 40 & 12,324 & 9 & 0,196 \\
\hline & Dependencia & 40 & 19,789 & 18 & 0,345 \\
\hline & Movilidad & 40 & 13,305 & 18 & 0,773 \\
\hline
\end{tabular}

Finalmente, se puede completar el análisis afirmando que las personas mayores disponen de una imagen positiva de este colectivo en la publicidad televisiva; del total de la muestra, un $45 \%$ incluye el adjetivo de "alegres" y un 17,5\% "trabajador" e "independiente" cuando describen a la persona mayor que aparece en un anuncio televisivo.

\section{- DISCUSIÓN Y CONCLUSIONES}

El factor de la institucionalización, como se exponía en el apartado anterior, supone ser crucial en la determinación de la percepción que tienen los dos subgrupos sobre el colectivo de personas mayores, mostrando así una autovaloración más positiva el subgrupo de personas mayores no institucionalizadas. Posiblemente, la diferencia perceptiva se deba a factores como una mayor autonomía o el mayor contacto con familiares, manifestando, de esta manera, que el hecho de envejecer no supone ningún impedimento para seguir disfrutando o para estar activo; de hecho, como se demuestra en la H4, las personas institucionalizadas manifiestan no poder realizar todas las actividades que les gustaría por el hecho de vivir en residencias, conclusión que puede vincularse con referencias en personas mayores institucionalizadas, donde se afirma que la calidad de vida de una persona no se ve asegurada por el hecho de vivir en una residencia, sino que puede ser una experiencia segregadora, restrictiva y pobre ${ }^{23}$ o que las personas que viven institucionalizadas cuentan con menos recursos (familiares y comunitarios) que las personas que viven en sus domicilios, y que, además, las relaciones sociales que se establecen en los entornos institucionalizados no son elegidas, sino que ya vienen dadas, y pueden provocar muchas veces insatisfacción o resignación ${ }^{25}$.

Por otro lado, el estudio muestra que los individuos que actúan de manera independiente y que asumen responsabilidades en sus necesidades o en sus ABVD poseen un mayor sentimiento de autoeficacia $(\mathrm{H} 1)$, conclusión alineada con los resultados de otros estudios ${ }^{25}$. De hecho, se ha podido observar que las personas no institucionalizadas gozan de más independencia y autonomía, presentando juicios de valor más positivos y menos estereotipados; lo contrario que ocurre con las personas mayores institucionalizadas, aspecto que puede deberse al hecho de haber perdido el sentimiento de autoeficacia como personas que aún son capaces de aportar a los demás.

Las H2 (a mayor consumo televisivo más imagen estereotipada de la vejez) y la $\mathrm{H} 3$ (mayor consumo en personas institucionalizadas) no se han demostrado, es decir, que en nuestra muestra no han existido diferencias significativas entre mayor consumo e imagen más estereotipada del colectivo de personas mayores ni diferencias significativas entre el número de horas de consumo televisivo entre personas institucionalizadas o no institucionalizadas.

Como limitaciones del estudio, las autoras quieren señalar que se trabaja con una muestra de 40 personas (20 institucionalizadas y 20 no institucionalizadas), si bien la muestra podría ampliarse a través de proyectos con financiamiento o mantenerse el estudio con dicha muestra (barrio e institución) de forma longitudinal a través de convenios.

Para finalizar, cabe señalar que este trabajo también incluye, de manera transversal, el estudio de la calidad de vida en las personas mayores, concluyendo que la pérdida de calidad de vida la encontramos en la presencia de la dependencia, la cual radica en no poder vivir de manera autónoma y necesitar de forma permanente la ayuda de otros para realizar las actividades de la vida cuotidiana ${ }^{6}$

\section{Conflicto de intereses}

Las autoras declaran no tener ningún conflicto de intereses relacionado con este artículo.

\section{Agradecimientos}

Las autoras agradecen la colaboración de las instituciones de la residencia de referencia en Baleares, Llar d'Ancians, así como los centros de día de Casal del Jonquet y Casal de la Soledat.

\section{- BIBLIOGRAFÍA}

1. Andrés S, Maestro R. Análisis crítico del discurso publicitario institucional/comercial sobre las personas mayores en España. Comunicar. 2014;21(XXII)189-97.

2. Ramos-Soler I, Mancebo-Aracil JF. La investigación sobre personas mayores y publicidad: análisis metodológico (1976-2012). Estudios sobre el Mensaje Periodístico. 2013;19:945-52.

3. Maestro RL. Representaciones sociales de las personas mayores en la publicidad. Análisis de anuncios en prensa no diaria en España ente los años 1980-2010. [Tesis doctoral]. Valladolid: Universidad de Valladolid; 2013.

4. Ors A Maciá L. Mayores institucionalizados. Valoración de la satisfacción y el bienestar en una residencia geriátrica religiosa. Gerokomos. 2013;24(1):18-21.
5. González A. Intervención en calidad de vida de personas mayores en la residencia San Sebastián [Trabajo final de Experto Universitario]. Madrid: UNED; 2013

6. Fernández JJ. Determinantes de la calidad de vida percibida por los ancianos de una residencia de tercera edad en dos contextos socioculturales diferentes, España y Cuba [Tesis doctoral]. Valencia: Universidad de Valencia; 2009

7. Östlund B. Watching television in later life: a deeper understanding of TV viewing in the homes of old people and in geriatric care contexts. Scand J Caring Sci. 2010;24(2):233-43.

8. Freixas A. La mires como la mires no la versa. Comunicación \& Cultura. 1998;3:29-40.
9. Ministerio de Trabajo y Política Social. Informe del Libro Blanco del Envejecimiento Activo. Madrid: IMSERSO; 2010.

10. Molina C, Meléndez JC, Navarro E Bienestar y calidad de vida en ancianos institucionalizados y no institucionalizados. Anal. psicología. 2008;24(2):312-9

11. Baumann S, Laat K. Aspiration and Compromise. Portrayals of Olde Adults in Television Advertising. En: Lee Harrintong C, Bielby D, Bardo AR, eds. Aging, Media and Culture. Londres: Lexintong Books; 2014. p. 13-24.

12. Martínez-Salanova E. La publicidad y su integración en las aulas. Comunicar. 1994:1(3):65-73.

13. Warren L, Maltby T, Cook J. Older Women's Lives and Voices: Participation and Policy in Sheffield. Research: Findings: 21. From the 
Growing Older Programme. Findings 21 [Internet]. Sheffield: University of Sheffield (UK); 2003 [citado 12 de marzo de 2018]. Disponible en: http://www.growingolder.group.shef.ac.uk/Go Findings_21.pdf

14. Aznar H, Álvarez Villa A, Suay Madrid A. Estudio sobre la contribución de la radio al envejecimiento activo. Fase inicial: análisis cuantitativo de la presencia de la vejez en los magazines de radio. Estudios sobre el Mensaje Periodístico. 2016;22(1):143-62.

15. Hersroni A. Advertising and reality. A Global Study of Representation and Content. Londres: Continuum International Publising Group; 2012

16. Díaz L. La imagen de las personas mayores en los medios de comunicación. Sociedad y Utopía. Revista de Ciencias Sociales. 2013:41:483-502

17. Ribera JM. Imagen de las personas mayores y medios de comunicación. En: Causapié P. Balbotín A, Mateo A, eds. Envejecimiento Activo. Libro Blanco. Madrid: IMSERSO; 2011. p. 353-369.
18. Ramos I. Los mayores, el consumo y comunicación publicitaria. El estilo de vida de las personas mayores y la comunicación publicitaria. Un análisis empírico. [Tesis doctoral]. Alicante: Universidad de Alicante; 2005

19. Kessler EM, Schwender C, Bowen CE. The Portrayal of Olde People's Social Participation on German Prime-Time TV Ad vertisements. J Gerontol B Psychol Sci Soc Sci. 2009;1:97106

20. Losada A. Edadismo: consecuencias de los estereotipos, del prejuicio y la discriminación en la atención a las personas mayores. Algunas pautas para la intervención. [Informes]. IMSERSO; 2004. Informes Portal Mayores, 14 [acceso 11 de marzo de 2016]. Disponible en: http://envejecimiento.csic.es/documentos/documentos/ losada-edadismo-01.pdf
21. Vasil L, Wass H. Portrayal of the elderly in the media: a literature review and implications for educational gerontologists. Educational Gerontology. 1993:19 (1):71-85.

22. Ramos I, Carretón MC. Presencia y representación de las personas mayores en la publicidad televisiva: el caso español. Rev Esp Geriat Gerontol. 2012;47(2):55-61.

23. Hurd C. We're Not Old!": Older Women's Negotiation of Aging and Oldness. J Aging Stud. 1999;13(4):419-39.

24. Fernández-Ballesteros R. Como quieren ser tratadas las personas mayores. En: Gómez MP, Díaz P, coord. Guía práctica del buen trato a las personas mayores. Madrid: Sociedad Española de Geriatría y Gerontología; 2011; p. 15-24

25. Abad L. Diseño de programas de e-inclusión para alfabetización mediática de personas mayores. Comunicar. 2014;21(XXII):173-80. 